\title{
Socialité et émotions
}

\section{Gérald Berthoud}

\section{OpenEdition \\ Journals}

Édition électronique

URL : http://journals.openedition.org/ress/66

DOI : $10.4000 /$ ress.66

ISSN : 1663-4446

\section{Éditeur}

Librairie Droz

\section{Édition imprimée}

Date de publication : 1 mai 2009

Pagination : $57-71$

ISBN : 978-2-600-01303-1

ISSN : 0048-8046

Référence électronique

Gérald Berthoud, "Socialité et émotions », Revue européenne des sciences sociales [En ligne], XLVII-144 | 2009, mis en ligne le 01 mai 2012, consulté le 20 avril 2019. URL : http:// journals.openedition.org/ress/66 ; DOI : 10.4000/ress.66 


\section{Gérald BERTHOUD}

\section{SOCIALITÉ ET ÉMOTIONS}

Le regard porté sur les émotions dans leur rapport avec la rationalité est celui d'un représentant des sciences sociales. Un regard qui ne se limite pas à un territoire disciplinaire étroitement circonscrit. Au contraire, il s'agit de développer un point de vue aussi englobant que possible. Tendre vers un tel objectif implique d'abord de clarifier quelque peu une notion aussi familière que celle d' «émotion » et pourtant si malaisée à définir. En passant du sens commun au langage scientifique l'imprécision subsiste. La confusion même tend à l'emporter en raison d'un découpage disciplinaire, qui entraîne diverses manières, très souvent inconciliables, d'envisager les émotions.

Mais une telle diversité s'inscrit dans une tradition culturelle, qui structure notre vision du monde à partir d'une logique d'oppositions binaires. Rationalité et émotions sont ainsi largement perçues comme des notions antagonistes. La pensée savante, même la plus critique, doit nécessairement se référer à cette tradition. Dans ce cas, la figure normative de l'être humain est celle d'un individu rationnel dont la socialité serait purement contractualiste. Une manière de présupposer que l'être humain dans sa nature même ne serait pas social. Mais nombre de travaux relevant, entre autres, du domaine des neurosciences soumettent cette conception réductrice de la socialité à de fortes critiques. Ils défendent la vision d'un être humain dont la capacité à nouer des relations avec autrui serait localisée dans le cerveau.

Avec les sciences sociales, tout au moins pour une partie d'entre elles, cette vue neurobiologique du cerveau fait l'impasse sur la dimension institutionnelle du social. Part irréductible de la condition humaine, le social, comme système d'obligations et de significations, suppose du même coup un regard plus ouvert sur le thème des émotions. En particulier, ces dernières n'apparaissent plus comme de simples négations d'une forme ou l'autre de rationalité. Elles ne se présentent plus comme des phénomènes proprement corporels, mais remplissent des fonctions et sont chargées de sens dans des contextes sociaux spécifiques.

Ce sont ces différents points qu'il s'agit maintenant de développer.

\section{QU'EST-CE QUE LES ÉMOTIONS?}

Nul doute que le terme «émotion» est largement usité dans le langage courant. Il est, par exemple, bien présent chez les journalistes. Il permet d'établir une distinction implicite ou non avec la froideur de la raison. Il évoque ce qui touche les êtres humains au plus profond d'eux-mêmes et qui s'extériorise de manière 
intense, jusqu'à se perdre dans l'irrationalité. Terme flou, l'émotion, qui se rapporte à des sensations soudaines et incontrôlables, permet de suggérer ce qui est en opposition plus ou moins radicale avec la raison. Il est, par exemple, déconseillé d'agir sous le coup d'une émotion, même si cette dernière nous surprend et nous envahit au point de nous submerger. La consultation des dictionnaires de langue confirme l'imprécision et l'ambiguité de l'usage courant. L'émotion et les termes de la même famille, émoi, émotif, émotionnel, émotivité, ou émouvoir, caractérisent tous une agitation, une effervescence, ou encore un trouble, qui se réfèrent à quelque chose d'agréable ou de désagréable. Ils peuvent se rapporter à une multitude de phénomènes et de situations au niveau individuel, interpersonnel et collectif. Des actions et des réactions, des paroles, des discours et des débats peuvent être caractérisés par un contenu émotionnel plus ou moins intense. $\mathrm{Ou}$ encore, des états émotionnels peuvent résulter d'événements dramatiques vécus directement ou non, en particulier par l'intermédiaire des médias. Dans tous les cas, s'opposent à émotion, à la fois calme, froideur, indifférence et insensibilité. En outre, dans un sens élargi ou affaibli, le terme émotion tend à se confondre avec ceux de sensation et de sentiment.

En passant du sens commun au langage scientifique, l'imprécision subsiste, même à l'intérieur d'un univers disciplinaire défini. Plus encore, l'idée d'émotion et les autres notions plus ou moins proches sont difficiles voire impossibles à distinguer. Elles ne permettent guère de séparer les différents moments d'un processus. Ce sont autant d'éléments constitutifs d'une nébuleuse sémantique, liés entre eux de manière floue et diffuse, ou encore d'un univers de sens plus ou moins confus. Des termes comme émotion, sentiments, humeur, affect, passion, mais aussi sensation, motivation, désir, ou encore pulsion ne se confondent pas assurément, mais ils ne sont pas clairement délimités. Fondamentalement ils ne peuvent pas l'être, tant ils sont étroitement liés. A consulter des dictionnaires spécialisés, il apparaît que la relation entre plusieurs de ces termes est celle d'un renvoi réciproque. Ce qui est particulièrement évident pour les trois termes d'émotion, de passion et de sentiment. Pour un lecteur qui consulterait successivement les différents termes de cette nébuleuse affective, il serait dans l'impossibilité d'établir des distinctions en raison d'un indéniable chevauchement des définitions. Il se heurterait à une certaine incohérence, en raison, entre autres, d'une simple juxtaposition des différents sens d'un même terme, au point de permettre une oscillation sans fin entre un terme et un autre ${ }^{1}$.

Dans de nombreuses publications, cette difficulté est en quelque sorte résolue en se centrant sur l'idée d'émotion, sans la moindre mention d'autres notions plus ou moins proches. Une manière d'envisager l'émotion comme une catégorie englobante. Cette façon de voir se retrouve dans les diverses tentatives d'établir des listes de phénomènes affectifs tous vus comme des émotions au sens large ${ }^{2}$.

1 Il serait certes possible, sinon nécessaire, d'établir des distinctions entre les trois termes de sentiment, d'émotion et de passion. Ils pourraient être çonçus comme des moments particuliers d'un même processus. Ou encore, il serait possible d'isoler des critères spécifiques comme l'intériorité pour les sentiments, la relative brièveté et la plus ou moins grande force d'une émotion ressentie et extériorisée, ou la force excessive et durable des passions.

2 Ces listes présentent d'évidentes divergences dans leur composition, à l'exclusion des émotions dites de base comme, par exemple, la peur, la colère, la tristesse, le dégoût, ou la joie. 
Mais cette diversité et cette confusion sont plus apparentes que réelles. Les émotions en général sont perçues et conçues à partir d'un noyau culturel constitutif du sens commun et de la pensée savante. Le thème des émotions, vu ainsi dans les limites de la tradition culturelle de l'Occident moderne, prend tout son sens à partir d'une logique d'oppositions binaires. Parmi les nombreuses oppositions constitutives d'une telle logique, celle de la rationalité et des émotions est largement attestée. La rationalité, comprise à la fois dans sa dimension instrumentale et discursive ou argumentative, est une notion-clé pour comprendre la place, la fonction et la signification variables des émotions selon les perspectives théoriques envisagées. C'est dire que toute approche argumentée doit partir du fait que les émotions et la rationalité sont culturellement vues comme des notions antagonistes. Avec un tel dualisme, les émotions représentent en quelque sorte la part d'irrationalité de tout être humain ${ }^{3}$. Elles apparaissent comme autant d'obstacles à la raison. Elles sont encore de simples négations de la rationalité vue comme un donné évident et indiscutable.

\section{LES ÉMOTIONS COMME «RESTE»}

Par exemple, les théories du choix rationnel (TCR) en arrivent tout simplement à ignorer le thème des émotions. Au mieux, les émotions apparaissent comme des «restes» empiriques ou des épiphénomènes. Elles ne sont que des entraves ou des obstacles que tout être humain jugé normal serait censé surmonter. Telle est la figure de l'homme rationnel centré sur ses propres intérêts. Pour le raisonnement économique standard, la règle est d'éviter de se perdre dans la complexité de la réalité des phénomènes humains et sociaux. Se confronter directement à une telle complexité entraînerait une confusion des idées. Dans cette perspective, la TCR, par sa relative simplicité, est sans équivalent. Pour elle, les motivations qui poussent les êtres humains à agir se réduisent à une composante rationnelle, vue comme une optimisation de l'intérêt individuel. Etre rationnel revient ainsi à rechercher le plus grand gain ou le plus grand avantage en toute situation pour la moindre dépense possible. Ainsi modélisé, tout individu incarnerait la tradition utilitariste du calcul du plaisir et des peines. Certes l'homo oeconomicus ainsi esquissé ne serait pas à la recherche exclusive de son bien-être matériel. Ses propres intérêts ne se résumeraient pas à accroître sans cesse ses gains monétaires ${ }^{4}$. Avec une représentation aussi réductrice de l'action humaine en général, la relation à autrui et plus largement le lien social ne peuvent être conçus que selon un point de vue strictement contractualiste. La socialité équivaut

3 Pour le sens commun, une croyance, qui est loin d'avoir disparu, établit que la rationalité s'oppose à l'émotion, comme l'homme s'oppose à la femme et à l'enfant, ou l'Occident dans sa globalité aux sociétés autres.

4 Pour Stigler, par exemple, lauréat du prix Nobel d'économie en 1983, «le concept de l'Homo oeconomicus ne sous-entend pas (comme le prétendent presque tous ses détracteurs) que l'individu cherche à maximiser argent et richesse, que l'âme humaine est un tiroir-caisse complexe. La théorie formelle n'est nullement ébranlée[...] parce qu'un individu maximise ses richesses ou sa piété ou ses succès divers, ou veille à sa ligne, etc.» (1946: 63-4). 
à une interdépendance fonctionnelle entre des individus vus comme indépendants. La forme émergente du marché, jusqu'à englober la totalité de relations sociales, constituerait la seule manière de tenir ensemble des êtres préoccupés par la recherche constante de leurs avantages. L'horizon idéal du raisonnement économique n'est-il pas celui d'une coordination grâce au jeu des prix de toute chose? N'est-il pas encore celui d'un monde dans lequel tout est quantifiable et mesurable? Nul doute que dans une représentation aussi réduite de la vie humaine, les émotions n'aient aucune place.

Mais la TCR n'est pas la seule approche rationaliste qui tend à se débarrasser, d'une manière ou d'une autre, de la question des émotions. Prenons à titre d'exemple le programme pour une «théorie sociologique générale», tel qu'il est présenté par le sociologue Boudon dans plusieurs articles et ouvrages. Le thème des émotions n'entre pas vraiment dans un tel programme. Les émotions, vues comme un donné du sens commun, n'auraient au mieux qu'une valeur résiduelle. Aussi Boudon déplore-t-il que « la mode est à l'affectif, à l'émotionnel et au culturel» (2008b: 158). Au contraire, son programme pour une sociologie proprement scientifique repose sur deux conditions:

- une théorie «individualiste» de l'action humaine;

- une conception élargie de la rationalité, selon une triple composante instrumentale, cognitive et axiologique (2008a: 46-47).

La scientificité de ce programme est ainsi fondée sur une représentation épurée de la réalité humaine et des phénomènes sociaux, en écartant tout ce qui est conçu comme des «forces occultes» internes et externes. Par soustraction en quelque sorte, il resterait la figure d'un homme dont les actions rationnelles porteraient sur les moyens et sur les fins. A partir de tels présupposés rationalistes, tout phénomène social serait le résultat d'une agrégation de croyances et de conduites individuelles. Cette manière de voir reposerait sur «des hypothèses microscopiques précises sur les raisons et les motivations inspirant le comportement des individus» (Boudon 2008a:37). Comment alors, dans une telle approche, concilier l'idée que «raisons et motivations » individuelles seraient la «cause ultime» et l'insistance sur la nécessaire prise en considération du contexte? En fait la théorie sociologique de Boudon est simultanément une conception de l'histoire vue comme une avancée progressive de la rationalisation, même si un tel mouvement n'est nullement constant et n'échappe guère à de possibles régressions. Un relatif optimisme pour lequel, au fil de l'histoire le «bon sens» l'emporterait contre les passions et les préjugés. Un point de vue qui repose sur l'hypothèse d'une sélection rationnelle des idées et des actions (voir Boudon 2008b:162). Dans une telle perspective évolutionniste, le prétendu primitif serait rationnel, même dans ses croyances et ses pratiques apparemment les moins conformes à la raison. Sa rationalité s'inscrirait dans les limites des institutions et des valeurs propres à son univers social. La diversité des sociétés et des cultures se réduirait ainsi à des phases successives dans un mouvement ascendant vers une rationalité effective, qui porterait à la fois sur les moyens et sur les fins. Dans cette tentative de combiner l'universel et le contextuel, les motifs de l'action ne devraient pas être purement internes. Ils supposeraient, implicitement tout au moins, la présence de la société en chaque individu, grâce à la socialisation et à l'éducation. Les moti- 
vations invoquées comme causes ultimes devraient nécessairement comprendre une part non explicitée d'un tout socialement institué. Il n'en reste pas moins qu'il y a chez Boudon une indéniable cohérence entre sa représentation d'un être humain foncièrement rationnel, son explication des phénomènes sociaux à partir des comportements individuels et sa conception évolutionniste de l'histoire. Il y a surtout une parfaite adéquation entre ses présupposés sur l'être humain et la part insignifiante attribuée aux émotions.

\section{CERVEAU ET ÉMOTIONS}

A l'inverse de la théorie du choix rationnel et du programme individualiste de Boudon, nombre d'auteurs conçoivent les émotions comme des éléments irréductibles pour comprendre les motivations, qui poussent les gens à prendre des décisions et à agir. Mais le thème des émotions, en vue de relativiser la portée d'une rationalité «froide», est en quelque sorte éclaté selon les limites disciplinaires propres aux domaines des sciences biologiques et sociales. Chaque discipline, selon ses programmes et ses points de vue, porte un regard spécifique sur les émotions, au point que leur complexité même échappe aux uns et aux autres. Il en résulte le plus souvent une juxtaposition d'analyses partielles et réductrices ${ }^{5}$.

Pourtant avec les développements considérables des neurosciences, la recherche sur les émotions semblerait échapper à une telle fragmentation. Le cerveau est vu comme un nouveau continent ${ }^{6}$. Son exploration au moyen des technologies les plus avancées devrait permettre une connaissance renouvelée de phénomènes psychiques comme la mémoire, la pensée, la langue, les représentations, la perception, ou encore les décisions, en vue d'en dégager les bases biologiques.

Il y a vingt-cinq ans déjà, Changeux, représentant notoire du nouvel horizon scientifique centré sur la neurobiologie, publiait L'homme neuronal. Pour lui, la biologie devenait le savoir par excellence sur l'homme et la société. Il devait permettre de «détruire les barrières qui séparent le neural du mental» (1983: 225). Aussi «l'homme n'a dès lors plus rien à faire de l''Esprit', il lui suffit d'être un Homme Neuronal» (1983: 227) ${ }^{7}$. Fort d'un tel savoir, Changeux met en garde

5 Pour la sociologie des émotions, par exemple, la structure sociale et les émotions constituent respectivement la variable indépendante et dépendante (voir Barbalet 2002: 3-4). Contre un tel découpage disciplinaire des émotions, le développement actuel des «sciences affectives » pourrait constituer une voie prometteuse (voir Sander et Scherer 2008 et le pôle de recherche national suisse en sciences affectives - http://www.affective-sciences.org/).

6 «Notre travail s'apparente à celui des cartographes à l'époque des explorateurs » affirme un chercheur en neurosciences (voir Reflex, publication de l'Ecole polytechnique fédérale de Lausanne, no2, 2007, p. 82).

7 Changeux affirme encore: «Pour le neurobiologiste que je suis, il est naturel de considérer que toute activité mentale, quelle qu'elle soit, réflexion ou décision, émotion ou sentiment, conscience de soi[...] est déterminée par l'ensemble des influx nerveux circulant dans des ensembles définis de cellules nerveuses, en réponse ou non à des signaux extérieurs. J'irai même plus loin en disant qu'elle n'est que cela» (voir le journal Le Monde 31.10.1982). Quinze plus tard Changeux n'a guère modifié sont point de vue naturaliste (voir Changeux et Ricoeur 1998). 
les représentants des sciences humaines et sociales contre une simplification portée à «trancher les liens profonds qui unissent le social au cérébral», mais ajoute-t-il, «plutôt que d'aborder le problème de front, on préfère, une fois de plus, occulter ce dangereux organe. Alors, décérébrons le social» (1983:3).

Depuis lors l'influence des neurosciences, sur les savoirs sur l'être humain comme individu et comme acteur social, n'a fait que croître. Cette manière de voir, centrée sur le cerveau, se vérifie, par exemple, dans la multiplication de termes construits à partir de l'élément «neuro». Faudrait-il ainsi élargir le champ des neurosciences, pour y inclure un ensemble de domaines toujours plus nombreux. La neuropsychologie et la neuroéconomie sont déjà largement reconnues. D'autres approches ont une visibilité moindre. Telles sont la neurophilosophie (voir, par exemple, Andrieu 2007 et Churchland 1999), ou encore la neuroéthique, la neuropédagogie et la neurothéologie vue comme une biologie des croyances religieuses. D'autres encore n'ont certes pas d'existence formelle pour le moment. Mais la voie semble ouverte pour une «neuroanthropologie» et une «neurosociologie». A moins d'envisager la disparition des sciences du social comme telles, au profit d'une social neuroscience. De toute évidence, une vision neuroscientifique du monde s'impose de plus en plus. Dans un entretien à la suite d'un colloque sur le thème «neurobiologie des valeurs humaines », Changeux affirmait: "Les neurosciences vont nous apporter une nouvelle vision, une nouvelle conception de l'homme et de l'humanité[...]. Les neurosciences inaugurent les Lumières du $\mathrm{XXI}^{\mathrm{e}}$ siècle ${ }^{8}$. A n'en pas douter, cette vision cérébralisée de l'être humain et du monde s'appuie sur des compétences technoscientifiques parmi les plus avancées, grâce, entre autres, à des technologies de pointe comme l'imagerie fonctionnelle par résonance magnétique (IRMf), ou la stimulation magnétique transcrânienne (SMT) ${ }^{9}$.

Les techniques de la neuro-imagerie, liées à diverses expérimentations comme le jeu de l'ultimatum ou du dictateur, sont largement présentes dans les domaines économique et financier, même si un grand nombre d'économistes ne sont en aucun cas des adeptes de ces techniques ${ }^{10}$. Certes la portée de la neuroéconomie est à première vue limitée, en se centrant sur les décisions individuelles et les choix des agents économiques. Mais pour les adeptes de cette approche, il devient possible, à partir de la visualisation du fonctionnement du cerveau, d'apporter une réponse rigoureuse à la question suivante: comment les individus prennent-ils des décisions et font-ils des choix? Faut-il alors penser que «le mérite de la

8 Voir Le Monde 02.02.2005.

9 L'IRMf est un procédé de visualisation des variations de flux sanguins dans les différentes parties du cerveau. Les images ainsi obtenues permettent d'analyser les connexions neuronales d'un individu en train de prendre une décision ou d'accomplir une tâche. Elles montrent les régions cérébrales activées par des émotions ou par un raisonnement. La SMT permet d'expérimenter les modifications d'un comportement quand une partie du cerveau est inhibée, au point de produire une lésion virtuelle, pour la durée de l'expérience, par des impulsions électriques. Pour un exemple de ce procédé, voir Knoch et al (2008).

10 «Une approche séductrice mais déjà contestée» titrait le journal Le Monde (15.01.08), en présentant, entre autres, un symposium à la New York University sur le thème général: Neuroeconomics: Decision making and the Brain. Le logo de la plaquette pour annoncer cette rencontre représentait une balance, avec un cerveau sur un plateau et le $\mathrm{S}$ barré du dollar sur l'autre. 
neuroéconomie est d'avoir démontré scientifiquement que l'émotion jouait un rôle aussi important que la rationalité dans la prise de décision ${ }^{11}$. A partir de l'imagerie cérébrale, la neuroéconomie et la neurofinance seraient donc en mesure de faire un tri entre les «bonnes» et les «mauvaises» émotions, ou plus rigoureusement de nous dire à quelles conditions, ou dans quelles situations, une émotion particulière présente une dimension fonctionnelle, ou, au contraire, une portée destructrice. L'homme rationnel pourrait ainsi instrumentaliser ses émotions pour accroître ses gains, en contrôlant, entre autres, tout désordre émotionnel conduisant à faire de mauvais choix.

Avec une telle démarche, la théorie du choix rationnel est soumise à une critique radicale. Cette théorie, constitutive du savoir économique orthodoxe ${ }^{12}$ et qui s'est imposée dans l'ensemble des sciences sociales, est vue toujours davantage comme une manière non réaliste de concevoir les prises de décision et les choix. Pour le sens commun rien de plus évident que les sujets humains soient guidés en partie par les émotions dans leurs actions comme dans leurs idées. Il en va de même pour toute une pensée savante hétérodoxe depuis longtemps. Telle est, par exemple, la position de l'école sociologique de Durkheim, dans la première moitié du $\mathrm{XX}^{\mathrm{e}}$ siècle. Contre la vision simplificatrice de l'homme centré sur ses propres intérêts, cette école défend l'image d'un être foncièrement ambivalent (voir Cuin 2001). D'une certaine manière, le neuroéconomie, en se servant des applications neuroscientifiques de l'imagerie cérébrale, ne fait que reprendre ces vues non-conformistes. Mais les moyens techniques utilisés sont radicalement nouveaux. Ce qui est pourtant bien insuffisant pour assurer la qualité scientifique d'une telle approche. En effet, les travaux se réclamant de la neuroéconomie et de la neurofinance présentent d'indéniables limites. En particulier, le contexte institutionnel tend à disparaître, ou il apparaît au mieux comme un donné. Il équivaut en quelque sorte à un ensemble de moyens à la disposition des individus. Une manière de voir qui s'exprime dans le langage de l'avoir, en privilégiant, souvent de manière exclusive, le rapport entre fins et moyens. Ce qui tend à naturaliser la représentation de l'individu indépendant, occupé en permanence à calculer ses gains et ses pertes. Une vision de l'humain aussi réductrice rend difficile, sinon impossible, toute réflexion sur la question du social.

Il n'en va pas de même avec les travaux relevant de ce qui passe pour la social neuroscience $e^{13}$. En particulier, à partir de l'examen de données neurobiologiques,

${ }^{11}$ Une position défendue par l'économiste français Christian Schmidt (voir Le Monde 15.01.08; voir également Gironde 2008). La même assurance est présente dans le domaine de la finance. Pour l'ingénierie financière, visant à optimiser les compétences des acteurs financiers, l'opposition traditionnelle entre émotion et rationalité est catégoriquement rejetée. Au contraire, «L'émotion pourrait même être un élément constitutif de la rationalité. Nous avons, par exemple, besoin d'un certain niveau d'émotion anticipée au moment de prendre des risques» (voir «La neurofinance réconcilie émotion et rationalité » dans Reflex, publication de l'Ecole polytechnique fédérale de Lausanne, no 4, 2008, p. 34).

${ }^{12}$ L'économiste Gary Becker, dans sa «Nobel Lecture» en 1992 affirme: «aucune approche d'une généralité comparable n'a été développée jusqu'ici qui présente une concurrence sérieuse pour la théorie du choix rationnel» $(1993: 402)$.

${ }^{13}$ La neuroscience dite sociale analyse les données fournies par les technologies d'imagerie cérébrale pour expliquer, entre autres, les comportements prosociaux, dans leurs aspects cognitifs et affectifs, comme l'altruisme et l'empathie, par exemple. Parrmi les nombreuses publications concernées, mentionnons Cacioppo et al (2003 et 2005) et Harmon-Jones (2007). 
tirées des techniques de la neuro-imagerie, la notion d'empathie, comme cette capacité individuelle de comprendre et de partager les sentiments et les émotions d'autrui, est clairement mise en évidence. Ainsi «les humains peuvent ressentir de l'empathie pour d'autres gens dans une très large variété de contextes pour des sensations et des émotions de base comme la colère, la peur, la tristesse, la joie, la douleur et le désir, aussi bien que pour des émotions plus complexes comme la culpabilité, l'embarras et l'amour» (Singer et Fehr 2005 : 341 ; voir aussi Vignemont et Singer 2006).

\section{LE PROBLÈME DU DEDANS ET DU DEHORS}

Avec une telle visibilité du fonctionnement du cerveau, tant sur le plan cognitif qu'affectif, faudrait-il en arriver à penser que tout est dans la tête? Nos relations avec les autres sont-elles en quelque sorte inscrites dans notre cerveau? Le point de vue neuroscientifique sur le social serait-il la seule manière d'en parler scientifiquement? Ou, bien plutôt, cette idée ne trouve-t-elle pas sa justification dans une culture portée à ramener la socialité à de simples relations intersubjectives?

Toutes ces questions et bien d'autres soulèvent un problème fondamental, celui de l'intériorité et de l'extériorité, ou du dedans et du dehors. La voie la plus facile et sans doute la plus répandue consiste à se centrer sur ce qui se passe à l'intérieur de l'individu, ou, au contraire, à l'extérieur, par exemple dans les pratiques individuelles et collectives. Avec l'approche neuroscientifique, pour expliquer les idées et les actions des êtres humains, la spécificité du contexte culturel et social est minimisée, voire ignorée. Les causes ultimes de tout phénomène humain seraient donc d'ordre physiologique et neurobiologique. En particulier, sentiments et émotions seraient des phénomènes foncièrement intérieurs ${ }^{14}$. Une telle centration sur le fonctionnement du cerveau comme la base majeure, sinon unique, des phénomènes affectifs et cognitifs n'évite que difficilement le piège d'un réductionnisme neurobiologique. Faut-il voir dans le lien établi entre la visualisation du fonctionnement du cerveau et une activité particulière en situation expérimentale un rapport de cause à effet? Mon cerveau serait-il ainsi la cause de mes décisions et de mes choix? Comment comprendre, par exemple, l'affirmation suivante:«le domaine nouveau de la neuroéconomie cherche à fonder la prise de décision économique dans le substrat biologique du cerveau» (Sanfey et al 2003: 1755)?

N'est-ce pas illusoire de rechercher un point de départ unique de toute chose? Ne serait-il pas plus prudent de poser une possible corrélation entre mon cerveau et mes motivations, mes actions, ou mes pensées, elles-mêmes en bonne partie soumises aux règles, aux normes et aux valeurs de mon univers social? A parcourir toute une littérature neuroscientifique, il est difficile de prendre position. Des expressions comme base neuronale, substrat neuronal et d'autres encore sont-elles des notions équivalentes à celle de cause? Sûrement pas. Et pourtant, pour un

${ }^{14}$ Ce point de vue est radicalement opposé à une tendance sociologique, tout aussi discutable, pour laquelle sentiments et émotions seraient socialement et culturellement construits. 
lecteur hors du champ neuroscientifique, une forte présomption de déterminisme causal est inévitable.

Mais à ces questions et ces doutes s'en ajoutent d'autres. En combinant les jeux expérimentaux, comme ceux de l'ultimatum et du dictateur, et les technologies d'imagerie cérébrale, l'expérimentateur peut voir ce qui se passe dans le cerveau d'un sujet placé dans une situation artificielle de choix et de prise de décision. Mais cette manière de simuler des aspects de la vie réelle ne va pas de soi. Le sujet d'expérience peut-il servir valablement de substitut d'un être humain, toujours pris dans un ensemble de relations à l'intérieur d'un contexte institutionnel donné? La réponse à cette question varie fortement selon les chercheurs. Sans surprise, ceux qui pratiquent de manière quasi exclusive les procédures expérimentales sont persuadés de leur supériorité par rapport à l'observation directe. Ils affirment une fois encore la préférence marquée pour la «rigueur» même au prix de la «simplification» (voir Esminger 2002: 60). Par exemple, Fehr, un des représentants notoires de ce courant, affirme: «Je procède comme en physique. Avant de décrire les relations économiques complexes qui peuvent s'installer entre deux individus, je cherche à établir des règles basiques, mais généralement valables ${ }^{15}$.

De plus, les données expérimentales et leur traduction sous la forme d'images cérébrales fonctionnelles ne sont pas des faits qui parlent d'eux-mêmes. L'expérimentateur doit interpréter ces images. Tenter ainsi de dégager le substrat cérébral pour expliquer les actions et les idées des sujets d'expérience est un point de vue, certes important, mais partiel, pour saisir l'être humain dans ses multiples composantes. Le risque d'une dérive interprétative, sous la forme d'un parti pris naturaliste ou tout au moins réductionniste, est grand. Les images produites par une technologie de pointe, comme plusieurs scientifiques le relèvent, captivent et présentent une apparence séduisante (voir Miller 2008 et Weisberg et al. 2008). Elles peuvent même être vues comme le produit d'une «nouvelle phrénologie» (voir Uttal 2001).

Certes, chaque domaine scientifique circonscrit son champ de recherche dans des limites relativement précises. Il en résulte un paysage disciplinaire fragmenté et potentiellement conflictuel. Chaque domaine se doit de délimiter son propre territoire; chaque discipline tend à se clore sur elle-même. Plus largement, le partage des tâches entre neurosciences et sciences sociales semble clair: aux premières, la dimension universelle des processus cognitifs et affectifs; aux secondes, les variations du comportement humain, ou encore la diversité sociale, culturelle et historique. Le thème des émotions illustre pleinement ce mouvement d'autonomisation disciplinaire. D'un côté, la voie naturaliste pour rendre compte d'une humanité commune; de l'autre, la voie culturaliste, jusqu'à s'enfermer dans une forme ou l'autre de relativisme. Ou encore, une humanité indifférenciée, pour l'une; des sociétés ou des cultures, pour l'autre. D'une part, les émotions sont vues fondamentalement comme des phénomènes biologiques et donc préculturels. Elles sont donc propres à l'espèce humaine, en étant conçues comme le résultat d'une

${ }^{15}$ Voir le journal Le Temps (30.09.2008). D'autres auteurs sont beaucoup moins affirmatifs. Ils relèvent à la fois les apports et les limites de l'expérimentation (voir Benz et Meier 2006; Gurven et Winking 2008). D'autres enfin, très sceptiques, ont de sérieux doutes sur la validité des résultats expérimentaux comparés aux comportements observés dans la vie réelle (voir Chibnik 2005). 
longue évolution sélective d'une humanité portée à accroître toujours plus son emprise sur le monde. D'autre part, la réalité des émotions résiderait dans la manière dont elles sont vécues dans un contexte social particulier. De ce point de vue, elles ne peuvent s'envisager qu'en relation étroite avec des croyances collectives, des valeurs sociales et du sens culturellement partagé. Nous sommes ainsi confrontés au double biais d'une interprétation relativiste et d'une interprétation naturaliste. Deux manières de s'enfermer dans le confort intellectuel de la spécialisation disciplinaire. Pour une science sociale ouverte, c'est-à-dire opposée à toute dérive relativiste et constructiviste, mais aussi à tout réductionnisme neurobiologique, le substrat cérébral conditionne l'être humain, mais il ne le détermine pas. Ce qui laisse à l'humanité présente et future un indéniable choix pour construire un monde commun ou pour s'autodétruire.

\section{LA NATURE DU SOCIAL}

A partir d'une telle présupposition, les émotions doivent être conçues comme autant de notions «transversales », renvoyant à l'opposition fondamentale entre une certaine permanence du biologique et la diversité des institutions sociales. Autant dire que les sciences sociales doivent, d'une façon ou d'une autre, se confronter aux positions naturalistes de la recherche neuroscientifique. L'idéal serait d'insister sur la part irréductible du social, tout en considérant effectivement le conditionnement biologique et les limites qu'il impose à toute vie humaine. Certes, personne n'est en mesure d'analyser une réalité humaine aussi contradictoire et ambivalente. Mais admettre une telle complexité, c'est rendre possible un dialogue entre neurosciences et sciences sociales. Pour aller dans une telle direction, mentionnons brièvement quelques points.

L'homme concret ou complet, tel est le point de départ ou le donné empirique des sciences sociales. Ce qui suppose nécessairement une représentation de l'être humain à partir de sa condition d'être vivant, c'est-à-dire caractérisé, selon toute une tradition savante bien attestée, par un ensemble de traits plus ou moins apparentés. Tels sont, en particulier, l'«instinct de conservation», ou l'«instinct de vie». Ou encore, l'amour de soi dans le langage de Rousseau et la pulsion de vie en termes freudiens. L'idée générale derrière ces formulations et bien d'autres encore est celle d'un être en mesure de se maintenir en vie grâce à ses capacités adaptatives. En d'autres termes, tout être vivant est vu comme une force, à la fois menaçante et menacée, dans ses affrontements inévitables avec les autres et contre les phénomènes naturels.

Mais cette vision d'une violence naturelle doit être relativisée. Comme nous l'apprennent avec insistance nombre de travaux en éthologie, il y a une socialité animale, en particulier chez les grands singes (voir, par exemple, Cheney et Seyfarth 1990, et De Waal et Tyack 2004). Il est ainsi possible de parler d'un «instinct social» ${ }^{16}$ propre à limiter la portée destructrice des besoins et des affects

${ }^{16}$ La notion d'instinct devrait être l'objet d'une analyse critique (voir de Waal 2006: 85). En particulier quand elle est associée à une vision foncièrement utilitaire de la vie humaine. Par exemple : «La sélection naturelle semble nous avoir fourni un instinct du pardon parce qu'il aidait nos ancêtres à préserver les relations qui avaient une utilité biologique» (McCullough 2008: 132). 
les plus élémentaires, tout au moins dans les limites d'un groupe défini. Tel est juste esquissé l'ancrage biologique qui limite nécessairement les multiples manières de vivre, élaborées par l'humanité à travers le temps et l'espace.

Il resterait alors à se demander ce qui est proprement humain, au point de nous différencier du monde animal, malgré l'évidence d'une base biologique commune. Un fond proprement anthropologique pourrait s'énoncer de manière succincte comme suit:l'être humain est faible et fragile; mais cet être fini et vulnérable est travaillé, de multiples manières, par des désirs insatiables de toutepuissance, par une constante envie de dépasser, imaginairement ou réellement, toute limite. Cette double caractérisation de l'humain exprime une contradiction anthropologique fondamentale entre la condition d'un être mortel et le désir d'immortalité. Cette contradiction suppose le besoin des autres, pour assurer l'existence individuelle et pour y donner un sens. Mais ce besoin des autres, même dans les relations les plus intimes, apparaît comme un jeu entre ce qui unit et ce qui sépare; il implique à la fois réciprocité et asymétrie.

Ainsi, à suivre Ricoeur: «l'action n'est pas seulement interaction, transaction, mettant en relation - et le plus souvent en conflit - une pluralité d'agents, mais relation asymétrique entre ce que l'un fait et ce qui est fait à l'autre, ce que l'autre subit. En ce sens, le problème moral naît de ce qu'une menace de violence est inhérente à la situation asymétrique de l'interaction; dès lors que quelqu'un exerce un pouvoir sur quelqu'un d'autre en agissant, la possibilité de traiter autrui comme un moyen et non comme une fin est inscrite dans la structure même de l'agir humain» (1994: 274).

Toutes les sociétés, passées, présentes et futures ne peuvent se concevoir, de manière réaliste, en dehors d'un tel fond anthropologique ${ }^{17}$. Partout et toujours, l'humanité, pour surmonter les contradictions de sa condition, est confrontée à la nécessité de trouver un équilibre nécessairement instable entre des exigences opposées. Les multiples réponses données par l'humanité, pour maîtriser une telle situation conflictuelle, ont pris chaque fois la forme d'un monde institué. La différence essentielle entre les êtres vivants humains et les autres doit s'établir à ce niveau. Pour nombre d'éthologistes, les notions de culture et de société sont transspécifiques, mais la singularité humaine résiderait dans une capacité de créations imaginaires, concrétisées sous la forme de cultures ou de sociétés dites symboliques. Certes seuls les individus pensent et agissent. Mais ils ne peuvent le faire qu'en se conformant plus ou moins à des lois, des règles et des normes propres à canaliser pulsions et affects, en vue d'imposer des façons de sentir, de penser d'agir et de maintenir une manière définie de vivre-ensemble. Mais la violence instituée et la force légitimée, nécessaires pour punir toute violation des lois, seraient bien insuffisantes pour assurer une vie sociale effective, ou pour établir un monde qui fonctionne tout en étant relativement cohérent. Une cohérence, pour quoi faire? Une question qui renvoie au sens de la vie humaine et à la finalité de l'existence individuelle et collective. Mais cette question en appelle immédiatement une autre. Quelle est la source de ce sens ou de cette finalité? L'individu vu normativement comme indépendant sinon autonome? Mais l'indi-

17 Sauf à suivre les prévisions des technoscientifiques les plus visionnaires pour lesquels le seul avenir possible serait celui d'une posthumanité. 
vidu, pris dans des contradictions existentielles, s'insère dans un contexte social et culturel légitimé par des principes normatifs.

Poser la question du social revient donc à poser la question du sens et en conséquence du pour quoi vivre et exister. Dans un contexte déterminé, l'individu est poussé à acquérir, de manière compétitive, ce qui est valorisé. Tels sont, par exemple, le prestige, l'honneur, la renommée, le pouvoir, ou encore la richesse. Ces fins valorisées constituent autant de modèles d'être, de penser et d'agir, qui indiquent ce qui est à faire et qui contribuent à la fabrication sociale des individus. Mais les fins valorisées d'une société donnée ne sont pas des moules imposant absolument leur forme aux individus. Il y a en tout être humain une «part maudite», ou un "part obscure» irréductible et jusqu'à un certain point socialement indomptable. Une telle part est repérable dans de multiples formes de transgression et d'excès. Ces tendances à la démesure entraînent des rivalités et des luttes, qui deviennent, dans des moments particuliers, des menaces plus ou moins graves pour la cohérence sociale à divers niveaux.

A partir d'une telle conception de la socialité, les émotions ne peuvent se réduire à la simplification d'un vécu intérieur, propre au sens commun individualiste. Elles présupposent un monde d'institutions comme un ensemble de règles d'action et d'idées constitutives, entre autres, de cette part impersonnelle de toute intériorité individuelle. Les émotions doivent donc être envisagées dans une perspective dynamique, en les considérant dans leurs diverses composantes. Il y a ainsi une genèse biopsychique des émotions, qui s'extériorisent dans des attitudes, des gestes et des paroles, et qui favorisent ou non la communication. Mais les institutions sociales permettent de canaliser les émotions en leur donnant un sens.

\section{UN ENJEU MAJEUR}

Pour conclure, le thème des émotions est révélateur d'un enjeu majeur entre deux manières de produire des connaissances scientifiques sur l'être humain et les sociétés. Deux champs disciplinaires se dégagent. D'une part, les neurosciences en rapport étroit avec la psychologie et l'économie; d'autre part, les sciences sociales, en particulier l'anthropologie culturelle et sociale et la sociologie. Les premières reposent sur l'idée d'une science à la recherche du niveau jugé le plus fondamental pour expliquer la complexité du social. Ce dernier, dans ses multiples aspects, serait vu comme une combinaison d'actions individuelles dont les motivations renverraient à des états mentaux, assimilés à une matière pensante résultant de processus biologiques constitutifs du cerveau.

Une telle succession linéaire est problématique pour les sciences sociales. Le contexte institutionnel, au sein duquel tout individu pense et agit, constitue un point de départ irréductible, même pour les démarches basées sur une conception individualiste du social. La socialité ne peut ainsi découler simplement de niveaux de moindre complexité. Les résultats tirés de la connaissance du fonctionnement du cerveau ne sont pas transposables sans autre pour comprendre l'émergence du social comme ordre institué.

Neurosciences dites sociales et sciences sociales partagent à première vue le même objet de recherche. Dans les deux cas, les conduites prosociales, comme l'altruisme ou l'empathie, sont mises en évidence contre la représentation domi- 
nante d'un être humain mû en toute situation par la recherche de ses intérêts individuels. Mais très immédiatement la question se pose de savoir en quoi l'un et l'autre champs disciplinaires relèvent du social. Deux points de vue sont aisément repérables. Pour le domaine neuroscientifique, l'adjectif social est subordonné à l'élément neuro. De manière ambiguë, le cerveau est posé comme la base ou le fondement des comportements sociaux. De manière extrême certes, il peut être vu comme la cause des interactions affectives, cognitives et du social comme tel. Pour une bonne part des sciences sociales, au contraire, toute relation humaine, avec les motivations et les émotions qui les constituent, ne s'explique que replacée dans un contexte social et culturel spécifique. Il y a donc, jusqu'à un certain point, un renversement de la relation entre ce qui est à expliquer et ce qui explique.

Dans les deux cas, mettre en évidence la composante biologique ou culturelle des émotions peut tout à fait se justifier, pour autant que l'une comme l'autre soient vues comme deux points de vue partiels. Mais la position la plus courante est de tendre vers une explication exclusive, fondée sur le cerveau ou, au contraire, sur un contexte institutionnel particulier. Neurosciences et sciences sociales, sauf exception, apparaissent ainsi comme deux univers scientifiques séparés, s'ignorant l'un l'autre et publiant dans des revues radicalement différentes. Mais les neurosciences, avec l'usage de technologies de pointe, possèdent un avantage majeur sur les sciences sociales. Elles peuvent donner l'impression de disposer de moyens inégalés pour une connaissance effective des phénomènes cognitifs et affectifs, en interprétant les connexions neuronales à partir des images cérébrales. Face à ce qui peut être perçu comme un «vrai réel», les sciences sociales sont soumises à des pressions internes et externes, en vue de se dégager des limites du sens commun. Perçues comme des «sciences molles», elles devraient «se durcir» selon des critères naturalistes, en vue de se constituer comme un savoir proprement scientifique. Confrontées à un tel défi, les sciences sociales peuvent certes revendiquer leur autonomie en restant dans les limites d'un ordre de réalité propre. Rien ne serait donné. Au contraire, tout serait construit, particulier, ou arbitraire. Tout devrait obéir à des lois sociales spécifiques et non pas à des lois de la nature ${ }^{18}$.

Une science sociale en mesure de considérer à la fois la dimension biologique de l'homme et sa capacité à créer des univers de sens supposerait de prendre en considération un certain nombre de points pour penser la condition humaine dans toute son ambivalence. Certes, le point de départ est celui d'un ensemble d'individus qui sentent, pensent et agissent dans un contexte institutionnel particulier. Mais le point d'ancrage d'une telle construction sociale réside nécessairement dans la nature biologique de l'homme. Cette capacité de créer des univers culturels multiples résulte d'une évolution biologique, qui pose ainsi des limites à l'imaginaire humain. Mais plus que de tenter d'expliciter cette capacité créatrice comme telle, il importerait de comprendre ces créations dans le temps et dans

${ }^{18}$ Les arguments des auteurs qui revendiquent explicitement l'autonomisation d'un savoir sur le social sont variables. A première vue, il semble pourtant possible d'envisager deux grandes tendances. D'une part, une approche à la fois relativiste, culturaliste et holiste; d'autre part, une approche universaliste, rationaliste et individualiste. Dans ce dernier cas, la conception de l'histoire est celle d'une rationalisation progressive de la vie humaine. 
l'espace comme autant de manières différentes d'orienter les individus vers des fins socialement valorisées. Une des tâches majeures des sciences sociales ne serait-elle pas de montrer que toutes ces fins ne se valent pas, sauf à retomber dans un relativisme normatif?

Tout ce qui précède doit être vu comme l'ébauche d'une approche relationnelle élargie de la socialité. A partir d'une perspective aussi englobante, le thème des émotions s'inscrit pleinement dans un ensemble de relations à la fois biologiques et sociales.

Université de Lausanne

\section{BIBLIOGRAPHIE}

Andrieu, Bernard, (2007). La neurophilosophie. Paris: PUF (Que sais-je?, $2^{\mathrm{e}}$ éd.).

Barbalet, Jack, (2002). «Introduction: why emotions are crucial». Dans Emotions and Sociology, J. Barbalet, éd., Oxford: Blackwell, pp. 1-9.

Becker, Gary, (1993). «Nobel Lecture: The Economic Way of Looking at Behavior ». Journal of Political Economy 3: 385-409.

Benz, Matthias et Stephan Meier, (2006).«Do People Behave in Experiments as in The Field? Evidence from Donations ». Institute for Empirical Research in Economics, University of Zürich: Working Paper no 248.

Boudon, Raymond, (2008a). «Mais où sont les théories générales d'antan?». Revue européenne des sciences sociales 140: 31-50.

- (2008b). «D'un Durkheim à l'autre». Dans Durkheim. L'institution de la sociologie, B. Valade, éd., Paris: PUF, pp. 151-171.

Cacioppo, John T., Lorig S. Tyler, Howard C. Nusbaum et Gary G. Berntson, (2003). «Social Neuroscience: Bridging Social and Biological Systems». Dans The Sage Handbook of Methods in Social Psychology, C. Sansone, C. C. Morf et A. T. Panter, eds. London: Sage, pp. 383-404.

Cacioppo, John T. et Gary G. Bernston, eds, (2005). Social Neuroscience: Key Readings. London: Psychology Press.

Changeux, Jean-Pierre, (1983). L'homme neuronal. Paris : Fayard.

Changeux, Jean-Pierre et Paul Ricoeur, (1998). Ce qui nous fait penser. La nature et les règles. Paris: Odile Jacob.

Cheney, Dorothy L. et Robert M. Seyfarth, (1990). How Monkeys See the World: Inside the Mind of Another Species. Chicago: University of Chicago Press.

Chibnik, Michael, (2005). «Experimental Economics in Anthropology: A Critical Assessment». American Ethnologist 32 (2):198-209.

Churchland, Patricia Smith, (1999). Neurophilosophie. Paris: PUF (première publication intitulée Neurophilosophy: toward a unified science of the mind-brain, MIT Press 1986).

Cuin, Charles-Henry, (2001). «Emotions et rationalité dans la sociologie classique: les cas de Weber et Dukheim». Revue européenne des sciences sociales 120: 77-99.

De Vignemont, Frédérique et Tania Singer, (2006). «The empathic brain: how, when and why». Trends in Cognitive Science 10 (10): 435-441.

De Waal, Frans, (2006). Le singe en nous. Paris: Fayard (première publication en anglais Our Inner Ape: the Best and the Worst of Human Nature, Granta Books 2005).

De Waal, Frans et Peter L. Tyack, eds, (2004). Animal Social Complexity: Intelligence, Culture, and Individualized Societies. Cambridge. Mass.: Harvard University Press.

Ehrenberg, Alain, (2008). «Le cerveau 'social'. Chimère épistémologique et vérité sociologique». Esprit 341: 79-103. 
Ensminger, Jean, (2002). «Experimental Economics: A Powerful New Method for Theory Testing in Anthropology». Dans Theory in Economic Anthropology. Jean Ensminger, ed., Walnut Creek CA: AltaMira Press, pp. 59-78.

Gironde, Sacha, (2008). La neuroéconomie. Comment le cerveau gère mes émotions. Paris: Plon.

Gurven, Michael et Jeffrey Winking, (2008). «Collective Action in Action: Prosocial Behavior in and out of the Laboratory ». American Anthropologist 110 (2): 179-190.

Harmon-Jones, Eddie et Piotr Winkielman, eds, (2007). Integrating Biological and Psychological Explanations of Social Behavior. New York: Guilford Press.

Knoch, Daria, Michael A.Nitsche, Urs Fishbacher, Christoph Eisenegger, Alavaro Pascual-Leone et Ernst Fehr, (2008). «Studying the Neurobiology of Social Interaction with Transcranial Direct Current Stimulation - The Example of Punishing Unfairness ». Cerebral Cortex 18: 1987-1990.

McCullough, Michael E., (2008). Beyond Revenge. The Evolution of the Forgiveness Instinct. San Francisco: Jossey-Bass.

Miller Greg, (2008). «Neuroimaging: Don’t Be Seduced by the Brain». Science 320: 1413.

Ricoeur, Paul, (1994). Lectures 3. Aux frontières de la philosophie. Paris: Seuil.

Sander, David et Klaus Scherer, eds, (2008). Oxford Companion of Affective Sciences. Oxford: Oxford University Press.

Sanfey, Alan G, James K. Rilling, Jessica A. Aronson, Leigh E. Nystrom, Jonathan D. Cohen, (2003). «The Neural Basis of Economic Decision-Making in the Ultimate Game». Science 300: 17551758 .

Singer, Tania et Ernst Fehr, (2005). «The Neuroeconomics of Mind Reading and Empathy ». American Economic Review 95 (2): 340-345.

Stigler, George, (1946). Theory of Price. New York: Macmillan.

Uttal, William R., (2001). The New Phrenology: The Limits of Localizing Cognitive Processes in the Brain. Cambridge, MA: MIT Press.

Weisberg, Deena S., Frank C. Keil, Joshua Goodstein, Elizabeth Rawson, and Jeremy R. Gray, (2008). «The Seductive Allure of Neuroscience Explanations». Journal of Cognitive Neuroscience 20 (3): $470-477$. 